\title{
Genomic Chaos Begets Psychiatric Disorder
}

\author{
Donard S. Dwyer \\ Departments of Psychiatry and Behavioral Medicine and Pharmacology, Toxicology and Neuroscience, \\ LSU Health Shreveport, Shreveport, LA, USA
}

\section{Keywords}

Genetics · Genomic chaos · Major depressive disorder ·

Schizophrenia - Spooky action-at-a-distance - Transposable elements $\cdot$ Trexons

\begin{abstract}
The processes that created the primordial genome are inextricably linked to current day vulnerability to developing a psychiatric disorder as summarized in this review article. Chaos and dynamic forces including duplication, transposition, and recombination generated the protogenome. To survive early stages of genome evolution, self-organization emerged to curb chaos. Eventually, the human genome evolved through a delicate balance of chaos/instability and organization/stability. However, recombination coldspots, silencing of transposable elements, and other measures to limit chaos also led to retention of variants that increase risk for disease. Moreover, ongoing dynamics in the genome creates various new mutations that determine liability for psychiatric disorders. Homologous recombination, long-range gene regulation, and gene interactions were all guided by spooky action-at-a-distance, which increased variability in the system. A probabilistic system of life was required to deal with a changing environment. This ensured the generation of outliers in the population, which enhanced the probabil-
\end{abstract}

ity that some members would survive unfavorable environmental impacts. Some of the outliers produced through this process in man are ill suited to cope with the complex demands of modern life. Genomic chaos and mental distress from the psychological challenges of modern living will inevitably converge to produce psychiatric disorders in man.

(c) 2020 S. Karger AG, Basel

\section{Introduction}

The human genome has been fine-tuned and optimized over thousands of years of evolution to specify humans and their behavior. However, it was born of chaos [1-3]. Likewise, the human brain is finely tuned to optimize complex input analysis and output selection. It too is founded on principles of chaos - stochastic versus directed opening of ion channels to alter neuronal firing rates, oscillations in node and network activity, explosive neuronal wiring followed by massive pruning, construction of probabilistic models of reality (sensation), and signal detection coupled to behavioral output [4-9]. Because these highly variable brain processes are specified by an equally dynamic genome, it is little wonder that in the case of psychiatric disorders this input-output system functions chaotically. For example, epigenetic regulation

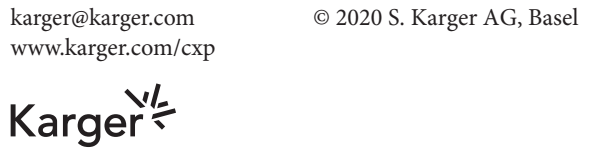

Dr. Donard S. Dwyer

Department of Psychiatry

LSU Health Sciences Center at Shreveport

1501 Kings Highway, Shreveport 71130 (USA)

ddwyer@lsuhsc.edu 
of ubiquitin-protein degradation systems may determine the rate of pruning in neurons, whereas stochastic expression of odorant receptors in particular neurons will affect odor sensation. Furthermore, too much pruning may alter the neuronal wiring needed for smooth integration of brain activity in distributed networks, and distortions during processing of sensory signals (misperception) may falsely lead someone to experience something that is not there (hallucinations). Consequently, there may be disorder in thought, emotions, social function, threat assessment, and cognitive ability.

Genetic factors are now well established as major contributors to the development of psychiatric disorders including schizophrenia, major depression, and bipolar disorder with heritability in the range of $\sim 50-80 \%$ [10$13]$. The fact that significant disability is associated with these disorders raises the question: how has this genetic liability emerged and persisted in the human genome? Intuitively (but perhaps over-simplistically), one would imagine that natural selection would limit the retention and transmission of genetic variants that contribute to such devastating disorders, especially when they typically first strike during prime reproductive years. There are many theories about why disease variants are found in the genome, including the idea of inevitable bad luck proposed by our group [14]; however, less has been written about how they arise and endure.

The evolution of the human genome - via random processes and counterbalancing adaptations to them created the conditions for eventual development of highly complicated nervous systems with the capacity for selfawareness, but also created conditions that inevitably lead to psychiatric disorder at some frequency in the population. The features of this chaotic system imprinted on man probabilistic behaviors with a wide range of normal function that are geared to cope with unpredictable input and shaping by the environment. When the behavioral output determined by various chaotic processes is suboptimum in several dimensions, psychiatric disorders can manifest. Here, I will summarize various connections that link disorder in the genome to disorder in the mind.

\section{Genetic Variation and Psychiatric Disorders}

Five main types of genetic variation can contribute to the risk of developing a psychiatric disorder. First, gene rearrangements, including translocations such as one involving disrupted in schizophrenia-1 (DISC1), were among the first genetic alterations associated with psychiatric disorders $[15,16]$. Second, copy number variants (CNVs) represent large gene regions that have either been deleted or duplicated to decrease or increase effective gene dosage $[17,18]$. Most arise from de novo mutations, but if present in an individual's germline cells, they can be passed to their offspring. Third, insertions or deletions (indels) are smaller compared to CNVs, but may nevertheless effectively disrupt or alter the function of the affected gene or regulatory element. Fourth, single-nucleotide polymorphisms (SNPs) have been identified as common disease risk factors [19-22]. They may occur in coding as well as noncoding sequences of genes including microRNA [23]. In certain instances, the SNP may produce nonsynonymous changes in the protein sequence leading to either loss-of-function or gain-of-function mutants. Most risk-gene SNPs are considered to have small genetic effect sizes and to be commonly expressed in the population. Some SNPs are associated with protective effects. Finally, somatic mutations may arise in neurons and other cell types causing tissue mosaicism for that genetic polymorphism $[24,25]$. Increasingly, it is becoming clear that such somatic mutations contribute to various disease states and degenerative conditions in man [25-27].

The comprehensive profile of genetic risk-gene variation that predisposes to psychiatric disorders constitutes the genetic architecture and includes the total number of risk variants, their genetic effect size, and frequency of occurrence in the population. Genome-wide association studies and theoretical models both suggest that variation in hundreds of common risk genes determines vulnerability to psychiatric disorder along with rarer CNVs or indels $[28,29]$. However, the contributions of CNVs, indels, and even somatic mutations occur on the background of many common SNP risk variants. Importantly, common risk variants must also be prevalent in the nonaffected population. In the case of schizophrenia, it is estimated that nonaffected individuals must carry $80-90 \%$ of the common risk variants required to reach threshold for disorder regardless of what that threshold level may be (M. Kasap and D. Dwyer, unpublished observations). Complex psychiatric disorders such as schizophrenia or major depressive disorder will require hits at many genes due, in part, to the number of different behavioral domains that must be adversely affected to cause sufficient deviation from the norm. 


\section{How Did We Get Here? The Genomic Chaos Machine}

At the "beginning" ... there must have been chaos without any functional organization among the immense variety of chemical species. Manfred Eigen [30]

The path to life began in a prebiotic soup of organic molecules synthesized haphazardly and arrayed randomly at the start [1]. Given the duality of nature, this state of chaos was counterbalanced by emerging organization [30]. The first step was self-organization with increasingly more complicated heteroatomic molecules aggregating and binding (like binds like [31]) to avoid destruction and to self-segregate from unlike others. Attraction between similar molecules via dipole interactions, hydrophobic effects, and complementation of delocalized orbitals fostered the emergence of self-catalysis [32] and/or template molding of building blocks to replicate monomers and then polymers, possibly explaining the origin of molecular replication. Molecular self-organization produced positive enthalpic changes (decrease in total free energy) and opposed entropy. By this model, the original bases and then nucleotides also self-organized to form short polymers. By folding back on themselves, most likely through early inverted repeat structures, the polymeric strands could resist hydrolysis, grow, and survive - an early case of prebiotic selection. Furthermore, inverted repeat regions, while offering protection against hydrolysis, created hotspots for strand breaks due to torsional stress on the polymer. These break points could then attract and react with similar remote sequences, which over time led to the evolution of transposable elements (TEs) with the ability to catalyze insertion into existing nucleotide strands.

Today, genetic liability for psychiatric disorders partly reflects this original process of genome construction. Formation of the initial protogenome relied on several major developments referenced above: (1) replication and duplication of segments of nucleotide polymers, (2) mobilization/insertion of TEs into existing polymers, which may have also served to repair damaged segments, and (3) an informative connection to coevolving molecules such as amino acids and proteins. Blalock and Smith [33] discovered that a codon and its antisense counterpart encode amino acids that are complementary and bind to each other suggesting iterative cross talk and modulation between these initially distinct systems. As this relationship advanced, we see evidence for the generation of duplication units - mobile segments of DNA (at this stage) called trexons (transposable exons) that specified short stretches of amino acids and served as building blocks for exons and beyond [34]. Previously, I described how this might have led to the evolution of peptide/protein ligands and their receptors and to RNA/DNA-binding proteins [34]. It is worth noting that retrotransposon-encoded proteins interact with the RNAs that encode them [35], which supports these earlier ideas.

TEs have been critical for protogenome expansion as judged by the fact that they collectively comprise $\sim 45 \%$ of the human genome [35-37] and include long interspersed element-1s (L1s), Alu sequences and other short interspersed elements, and endogenous retroviral sequences. Moreover, $\sim 25 \%$ of human promoter regions include TE-derived sequences [38]. Transposition and translocation remain active features of the human genome and are associated with schizophrenia, bipolar disorder, and obsessive-compulsive disorder [39, 40]. Continuous rounds of duplication of nucleotide sequences [41-43] and insertion of new ones (via transposition) rapidly enlarged the protogenome $[44,45]$, which became double stranded, folded, and associated with other molecules to resist degradation. Stochastic processes created additional sequences and diversity (mutations) within these sequences, which became increasingly adept at selfpreservation due to selective pressures. In this context, stochastic refers to chance or unpredictable events that altered the original DNA sequence via physicochemical (e.g., oxidative damage, ultraviolet or other radiation, or transposition) or enzymatic (the wrong base in a DNA polymerase catalytic site) mechanisms.

Homologous recombination emerged reflecting fundamental principles such as like binds like [31] and selfperpetuation via duplication $[46,47]$. It can be used for horizontal gene transfer, but chromosomal rearrangements may also separate different genes originally in close proximity or stitch them together from distal locations (e.g., syntenic blocks of genes; see below [47]). At some stage, the protogenome became instructive for protein formation, integrated with primitive energetics systems, and incorporated into membranous structures culminating in the appearance of autonomous cells. Features of this protogenomic chaos persist until today in the form of TEs, recombination, strand breaks and repair mechanisms, and deletions and inversions of highly evolved genetic material.

Early RNA-DNA polymer expansion was based on mobility (dynamic and probabilistic) and similarity (e.g., homologous recombination). High recombination rates (associated with increased mutability and instability) attenuated risk by decreasing survival of deleterious se-
Dwyer 
quences, but accelerated the spread of genetic material. Paradoxically, these same chaotic processes also created sequence diversity, which served to restrain homologous recombination at sites that diversified, thereby stabilizing polynucleotide tracts. Along similar lines, Kent et al. [48] reported that there was typically an inverse relationship between the presence of TEs in the genome and recombination frequency at those sites. Impreciseness and chance generated random changes in the genome and provided the substrates that were acted upon and selected during evolution. However, evolution also depended on the fixation or protection of advantageous changes that arose in the genome. Thus, there was an intrinsic yin-and-yang relationship between chaos/instability and organization/ stability of the system. One would not exist without the other.

\section{Curbing Chaos}

Although born of chaos, the genomes of the first living organisms soon came under pressure to curb these tendencies. Without this pushback, ongoing genetic mechanisms such as recombination and transposition of mobile elements would disrupt the precise nucleotide structures required to specify life or define a species. As discussed already, sequence diversification and stabilization against recombination curbed chaos, but other mechanisms - inversions and/or genetic rearrangements, methylation of bases, and interaction with proteins such as histones also contributed. Chromosomal rearrangement of genes or gene segments created a barrier against homologous recombination when the sequence was inverted or shuffled and nearby deletions occurred. Similar processes can preserve genomic sequences generated during evolution that define or demarcate a species [49]. In addition, excision of terminal repeats during transposition prevented the further spread of different mobile genetic elements to maintain the status quo.

DNA superstructure represents another mechanism for curbing genomic chaos. Tightly packed DNA shows less recombination and greater linkage disequilibrium [50]. Recombination coldspots are characterized by changes in base composition (low GC content) and the presence of special motifs $[50,51]$; they also coincide with topologically associated domains (TADs) $[52,53]$. Proximity to centromeres correlates with a higher degree of linkage disequilibrium, whereas proximity to telomeres (more fluid regions of a chromosome) shows the opposite tendency [50].

Genomic Chaos Begets Psychiatric Disorder
Sites in strong linkage disequilibrium preserve gene interactions and coregulation via long-range promoters/ enhancers but also potentially create safe harbors for risk variants [54-56]. By curbing chaos via recombination coldspots, Hill-Robertson interference, and intra- and interchromosomal interactions (see the next section), weakly to moderately deleterious mutations can be trapped in the genome or at least become difficult to remove [57]. Furthermore, the closer a gene (and its product) is to optimum functioning, as the result of millions of years of evolution, it increases the likelihood that any mutation will have a negative impact. Highly conserved and optimized genes will tend to gravitate toward or be swept up in regions of reduced recombination [55] or less mutable genomic microenvironments. Many small-effect risk variants would be expected to tag along, survive, and be propagated under these conditions.

Limiting chaotic changes in the genome would be important for maintaining pleiotropic variants that positively contribute to a favorable phenotype despite increasing susceptibility for a psychiatric disorder. This possibility is known as the balancing-selection hypothesis and explains the preservation of detrimental risk variants in the genome $[58,59]$. The potential contributions of pleiotropic variants reach their apex in omnigenic models of complex disease causation (e.g., see [60]). These models propose a set of core genes with the strongest association to disease-related phenotypes whose effects are modified by a very large number of small-effect risk variants distributed throughout the genome. Omnigenic regulatory effects of these variants are mediated by the natural interconnectedness of genes ([60]; also discussed below). These interconnections were built into the system during evolution through the emergence of trexons, duplication, recombination, and gene rearrangements [34]. Measures to curb chaos (e.g., reduced DNA accessibility) may be necessary to disengage these interconnections and prevent excessive diffusion of disease risk liability.

\section{Genomic Chaos and Genetic Liability}

In this section, I will briefly describe how genomic chaos generates the genetic liability associated with various psychiatric disorders. Figure 1 depicts the various chaotic processes that lead to genetic polymorphisms and confer risk for altered phenotypes and behavior. Two general processes are involved: (1) creation of genomic instability/mutability and (2) adaptive protection that restrains the elimination of risk alleles. Insertion of TEs can

Complex Psychiatry 2020;6:20-29 
Fig. 1. Genomic chaos generates genetic polymorphisms that increase the risk for psychiatric disorders. Various dynamic processes (outer ring), for example, TE insertion and homologous recombination, and inherent genomic features (SNPs) affect the function/expression of genes that determine phenotypes and behaviors associated with psychological wellbeing. When the collective adverse effects of genetic variation alter normal function in enough traits or symptom domains, disordered behavior can outwardly manifest. TE, transposable element; $\mathrm{CNV}$, copy number variant; SNP, single-nucleotide polymorphism; DSB, double-strand break.

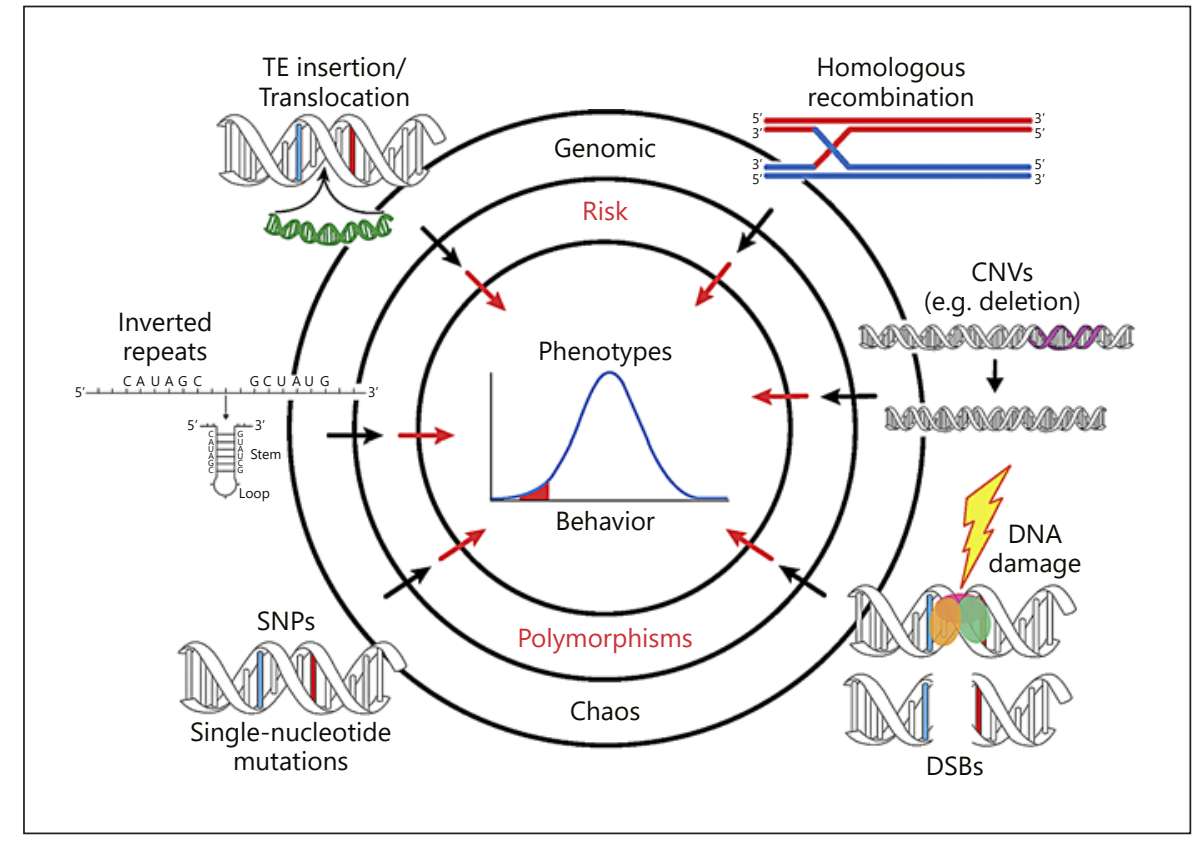

directly disrupt gene structure and expression including alteration of regulatory elements. In addition, insertion sites represent hotspots for future transposition, rearrangements, and mutation. Consequently, TE silencing has emerged as an adaptive protection, but it can spill over to protect nearby risk variants against removal [48]. Palindromes, inverted repeats, and repeat sequences affect local DNA structure and have been shown to cause instability and even promote double-strand breaks (DSBs) [44, $61,62]$. Palindromes, in particular, have been implicated in chromosomal translocations and 22q syndromes relevant for schizophrenia and bipolar disorder [44, 63]. Homologous recombination can disrupt or mutate a gene and lead to the emergence of recombination coldspots, which tend to preserve risk variants in the genome. Intriguingly, INO80E, a risk-gene candidate in schizophrenia [64], is involved in recombination and chromatin remodeling [46]. Repeat sequences in general (1) can be targeted during recombination, (2) represent sites of transposition $[61,65]$, and (3) often flank CNVs generated de novo [29]. Many stochastic processes cause somatic mutation (and parental germline mutation), but ultimately depend on a failure of DNA repair and fidelity [62]. Not surprisingly then, some risk variants associated with psychiatric disorders mediate DNA repair and damage responses $[14,66,67]$. Inaccurate DNA repair, fidelity issues during DNA replication, resolution of chemical modification of bases, and DSBs can contribute to the appearance of SNPS in the genome. Finally, epigenetic contributions couple environmental signals to regulation of gene expression and show connections to risk genes implicated in the pathogenesis of schizophrenia [14,68].

Risk variants with small genetic effect sizes will often survive selection via Hill-Robertson interference especially with low levels of recombination [54]. Indeed, Hussin et al. [55] and Pardiñas et al. [56] showed that low recombination regions of the genome are enriched for disease-associated variants. In addition, there are genomic hotspots for missense mutation that commonly generate variants implicated in neuronal function and neurodevelopmental disorders [69]. Somatic mutations are increasingly being appreciated as contributing factors to psychiatric conditions and neurological disease $[25,26]$. Recombination, palindromes, and interaction between topological domains can generate DSBs and mutations in neuropsychiatric disorders, other diseases, and cancer [70]. De novo retrotransposition (a directed chaotic process) alters gene expression patterns in the brain [71] and affects neuronal genomes of patients with schizophrenia [72]. L1 CNVs have been linked to somatic mosaicism in brain neurons [35]. Finally, it has been estimated that L1mediated retrotransposition may produce $\sim 0.1 \%$ of disease-producing insertions in man [73] on top of its more subtle effects on regulation of gene expression.

The delicate balance between chaos and organization is clearly manifested in gene-gene interactions. Epistasis and gene interaction networks provide functional organization, but act over considerable distances in some cas- 
Fig. 2. Epistatic ripple effects and amplification of genetic risk. Many risk polymorphisms implicated in psychiatric disorders have small genetic effect sizes individually (left side of the illustration). However, these small individual effects can be amplified when the polymorphism also affects the activity of nearby genes that are linked in a common purpose (syntenic blocks). Because genes operate in networks including epistatic interactions, the effects of genetic variation can ripple out to influence other genes in the network. Epigenetic regulation and/or chromatin accessibility can conceivably enhance or diminish this amplification of risk variants in the genome.

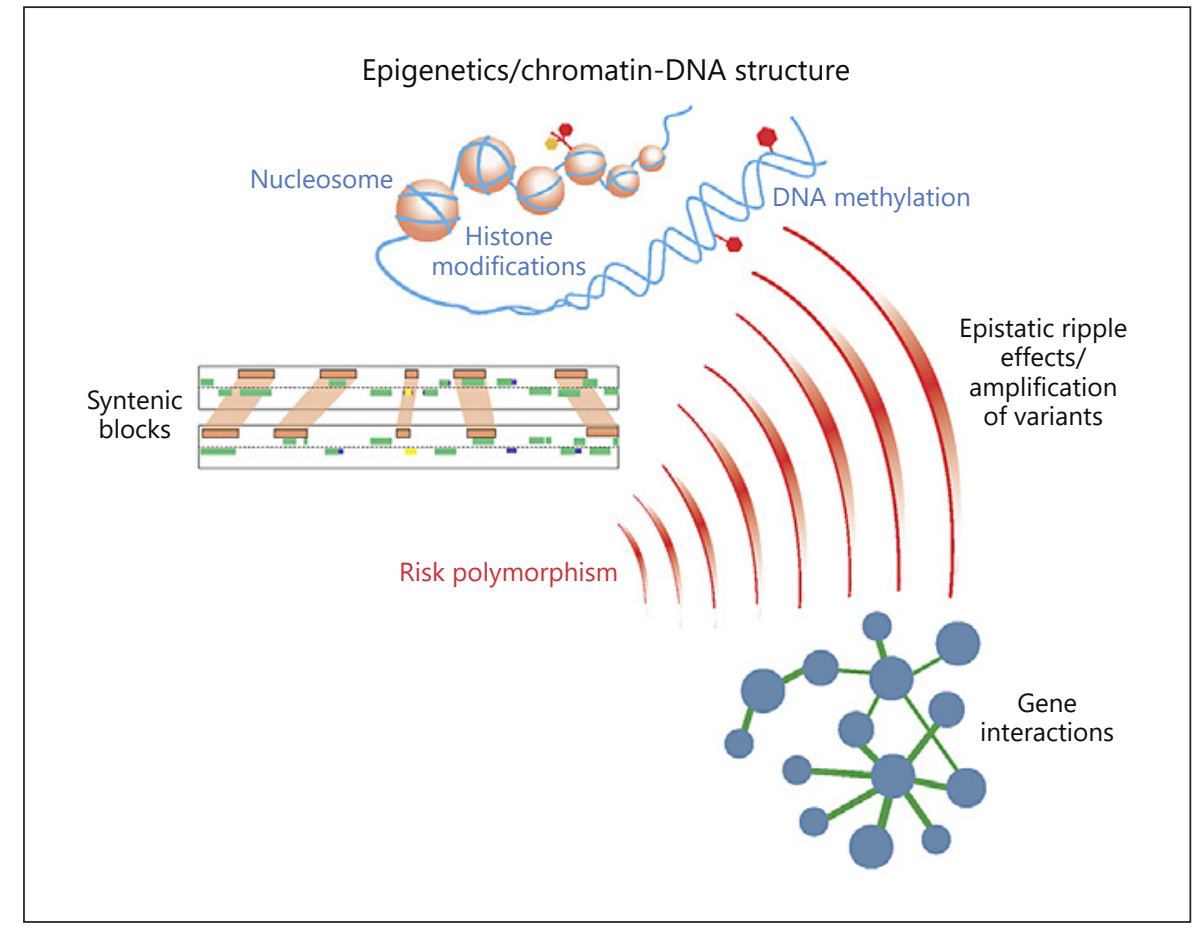

es and even between chromosomes (see the next section). Gene networks and genomic topology represent important sites for selection during evolution [74]. Nevertheless, boundaries of genomic domains are highly variable and interactions between distal chromatin segments are typified by stochasticity [75]. Furthermore, as the number of interacting genes increases so do the degrees of freedom of the system whose behavior is then much less predictable and more probabilistic. Gene-gene interactions amplify small genetic effect sizes of polymorphic risk variants and produce reverberation in networks referred to previously as epistatic ripple effects [76]. Figure 2 illustrates how gene interactions (local and long range) and epigenetic regulation can cause such ripple effects and amplification. Genetic interactions are an inevitable outcome of the way that the genome was constructed, but also spread risk when mutations are introduced into the system similar to the more rapid spread of infectious disease in highly interconnected populations.

\section{Gene Interactions, Syntenic Blocks of Genes, and Spooky Action-at-a-Distance}

During evolution, genes that mediated a common purpose frequently interacted with each other and, in some cases, rearranged into syntenic blocks of genes. Recently, syntenic blocks of functionally related genes have been implicated in schizophrenia [14]. Functional gene blocks not only colocalized over limited stretches of DNA but also experienced joint regulation over significant distances including interchromosomally [77]. How did these functionally related genes find each other over the vast expanse of the genome? Here, I refer to this long-range process as "spooky action-at-a-distance" after Albert Einstein's evocative description of quantum entanglement. Spooky action-at-a-distance is significant because it adds a powerful new dimension to gene regulation, DNA superstructure, and the opportunity for stochastic events to interfere with normal gene activity and cause disease.

The existence of long-range effects of regulatory elements across chromosomes has been known for some time including regulation of $\beta$-globin genes and the bithorax complex in Drosophila [78, 79]. Long-range interactions within chromosomes have been established as a mechanism controlling the 3-D structure of a chromosome [80] and the expression of linked genes, for example, TH2-cell-derived cytokines [81]. Lomvardas et al. [82] demonstrated that a single enhancer element could activate one odorant receptor allele on either the same or even a different chromosome. Functional interchromosomal contacts have been demonstrated in different ways [83] and mediate coregulated gene expression [84]. Won et al. [85] found that regulatory SNP variants do not nec- 
essarily control expression of the nearest gene; in fact, $65 \%$ of enhancers do not interact with the gene adjacent to them. On the other hand, some enhancers interact with genes $>500 \mathrm{~kb}$ away. In a similar vein, Whalen and Pollard [86] showed that chromatin contact frequencies correlated poorly with linkage disequilibrium maps, but did relate to levels of gene expression over significant distances. Together, these data suggest regulatory gene interactions may span long distances and simultaneously coordinate expression of multiple genes and gene blocks.

Gene amalgamation refers to the process of bringing together genes in syntenic blocks that function cooperatively or are regulated largely as a unit $[14,87,88]$. Integrons are a classic example of gene amalgamation whereby multiple genes involved in antibiotic resistance accumulated during evolution of bacteria [89]. The risk genes for schizophrenia include multiple syntenic blocks geared toward different biological purposes, for example, protein phosphorylation, transcriptional regulation, and DNA damage and repair [14]. Others [87] have noted the organization of genes into functional blocks mediating development, signal transduction, and transport processes, so this appears to be a widespread phenomenon.

The formation of syntenic blocks of genes was a natural outgrowth of genome dynamics. Because the genome was generated via extensive duplication, transposition, and homologous recombination, functional motifs such as promoter or enhancer regions were dispersed to multiple locations along with the genes they regulated. Divergence in the sequences of these duplicated segments led to generation of new genes with new functions. At the same time, convergence of functional activity between different genes could be jointly organized and regulated via their shared regulatory elements (promoters, enhancers, and others). Barzel and Kupiec [90] have shown that similar promoter regions normally located far apart in the genome can be organized into local "transcription factories" with close molecular contacts. This proximity meant that nonallelic homologous recombination between anciently dispersed duplications along with concerted movement via TEs could create syntenic blocks of functionally connected genes during evolution. Moreover, contacts between TADs based on functional connectivity could orchestrate reshuffling of genes during repair of nearby DSBs caused by torsional stress on the DNA backbone. Finally, compression of regions containing amalgamated genes can occur by deletion of repeat sequences, mobile elements, and transposition hotpot motifs to stabilize the new arrangements.

Interaction between TADs, the search for homologous chromosomal regions for recombination repair, long- range enhancers and promoters, and gene amalgamation are all guided by spooky action-at-a-distance. One can only speculate about the genomic features that mediate this activity. The 3-D structure of chromatin forms a distinctive landscape with low-resolution features that distinguish TADs and other subdomains and create recognition sites for DNA-binding proteins. Sequence motifs represent a higher resolution map of local features that can be searched and recognized. Ultimately, the electronic properties of nucleotide polymers and associated quantum attributes are likely to generate distinctive signals and reactivity (e.g., fast charge transfer along DNA creating appreciable electric fields) that can operate rapidly over significant distances [91, 92]. Propagation of selforganization - like binds like (association of DNA subdomains with similar features), alignment of dipoles in molecular electric fields, and $\pi$ interactions mediating noncovalent stacking of aromatic bases - to oppose chaos during evolution may have preserved a kind of molecular memory or pattern recognition system in DNA. Like any memory, formation of molecular memory in DNA during evolution would require an engram or substrate (a unique signature of electronic properties/quantum attributes), saliency or property (e.g., DNA deformability or flexibility, etc.), and a spatial dimension, for example, a $\mathrm{TAD}$ near the centromere of chromosome 2 . In addition, precise arrangement of repeat sequences, TE-derived segments, transcription factor binding sites, and other characteristic features in the genome left behind traces of their genesis along with variable local properties (e.g., deformability) that determine participation in particular processes, for example, recombination or transposition. This molecular memory may be responsible for spooky actionat-a-distance and adds another layer of complexity to biological systems.

Multidimensional regulation of gene output generates probabilistic outcomes (phenotypes and behavior) better suited to ensure the survival of a species because selection is then acting on traits that are normally distributed in the population. This also guarantees the emergence of outliers to increase the chance that some members of the population will survive an ever-changing environment. In addition, the environment can affect gene expression via different epigenetic processes such as DNA methylation. Interplay between the environment and DNA methylation has actually shaped the genome in ways we are just beginning to appreciate [93]. Because resolution of methylated cytosines can lead to gene mutations, DNA methylation provides a way to respond to environmental shifts with increased mutability and the potential for generating
Dwyer 
new beneficial variants. Furthermore, methylation of DNA in TEs can suppress their activity - yet another way to curb genomic chaos. There may be surprising findings ahead especially in the area of gene-environment interactions contributing to psychiatric disorders.

As discussed here, a probabilistic system of life was needed to counteract an unpredictable environment. Some of the outliers produced through this process in man are less equipped for dealing with the highly demanding modern environment, characterized by complex social interactions and significant occupational/financial requirements for survival. They represent our patients with psychiatric disorders.

\section{Conclusions and Perspectives}

Psychological wellbeing is a metastable state: a person can always feel better or worse than they do presently based on a shift in external circumstances or the internal milieu (e.g., physiological changes or neurotransmitter release). Consequently, a minor change in initial conditions can lead to very different outcomes via chaotic processes. The initial conditions of individual behavior are determined, in part, by genetic vulnerability (presence of heritable risk variants and de novo mutations), which is itself a function of genomic chaos as outlined here. In addition, more complicated behavior such as depressive symptoms or mania requires more genes to express and control it. Therefore, the genetic architecture of psychiatric disorders is correspondingly complex with perhaps hundreds of risk genes contributing. Many risk variants cannot easily be eliminated because they are pleiotropic with positive effects on other phenotypes and they are protected by mechanisms for curbing chaos (e.g., recombination coldspots and chromatin inaccessibility).

Probabilistic genomic behavior matches the environment to ensure survival, and it actually mirrors psychiatric disorders too. The genome and psychiatric disorders are both characterized by self-reiteration/perpetuation (duplication, transposition, and replication vs. addiction, obsessive-compulsive disorder, and learned depressive behavior) and self-disruption (recombination, mutation, and DSBs vs. schizophrenia, self-harm, and suicidality). In both cases, there is a yin-and-yang relationship between chaos and organization. For example, brain organization allows us to make sense of a cacophony of auditory signals during an operatic performance, whereas brain disorganization can create "voices in the head" of our patients with auditory hallucinations.
Inevitably, gene variants will arise in the genome that produce suboptimum behavior in various domains at some recurring frequency in the population. Given this inevitability, is it fruitful to consider these disorders to be illnesses? Rather, according to this model, psychiatric disorders are a product of dynamic genomes interacting with the environment to specify behaviors that are sometimes incompatible with the demands and pressures of modern living.

It is unlikely that we can fully undo what is a built-in feature of the biological system that is man. Importantly, altered neurochemistry is only one piece of the psychiatric puzzle. Therefore, our goal in long-term treatment (beyond short-term stabilization with medications) should be twofold: (1) to remove or limit environmental stressors and challenges that expose suboptimum behavioral strategies and (2) to help our patients realize that their chaotic inner worlds are a normal part of the natural (dis)order and not an aberration of it. With this mindset, we may learn to recognize the adaptive value of delusions (imagined refuge from threatening circumstances), manic behavior (bold or unpredictable actions taken against all odds), and even depression (conservation of behavioral output when the odds for success are low). Sharing of genetic liability across disorders, the normal distribution of normal/abnormal behavior, and chaos at the heart of it all should also encourage us to reexamine the rigidity of diagnostic categories and the dangers of pigeonholing our patients. As we appreciate the inevitability of disorder in biological systems, it will enhance our understanding of diversity and disorder in the human mind.

\section{Acknowledgements}

I thank Merve Kasap and Alex Cristino for invaluable comments and suggestions for the manuscript. I am also grateful to the Department of Psychiatry and Behavioral Medicine at LSU Health Shreveport for continuing support.

\section{Disclosure Statement}

The author has no conflicts of interest to declare.

\section{Funding Sources}

There were no outside sources of funding for this work. 


\section{References}

1 Orgel LE. The origin of life: a review of facts and speculations. Trends Biochem Sci. 1998; 23:491-5

2 Vinogradov AE. Evolution of genome size: multilevel selection, mutation bias or dynamical chaos? Curr Opin Genet Dev. 2004; 14:620-8.

3 Springer NM, Lisch D, Li Q. Creating order from chaos: epigenome dynamics in plants with complex genomes. Plant Cell. 2016; 28:314-25.

4 Jackson MB. Stochastic behavior of a manychannel membrane system. Biophys J. 1985; 47:129-37.

5 Korn H, Faure P. Is there chaos in the brain? II. Experimental evidence and related models. C R Biol. 2003;326:787-840.

6 Pouget A, Beck JM, Ma WJ, Latham PE. Probabilistic brains: knowns and unknowns. Nat Neurosci. 2013;16:1170-8.

7 Wierenga CJ. Live imaging of inhibitor axons: synapse formation as a dynamic trial-and-error process. Brain Res Bull. 2017;129:43-9.

8 Landau ID, Sompolinsky H. Coherent chaos in a recurrent neural network with structured connectivity. PLoS Comput Biol. 2018, 14:e1006309.

9 Nicola W, Hellyer PJ, Campbell SA, Clopath C. Chaos in homeostatically regulated neural systems. Chaos. 2018;28:083104.

10 Sullivan PF, Neale MC, Kendler KS. Genetic epidemiology of major depression: review and meta-analysis. Am J Psychiatry. 2000; 157:1552-62.

11 Smoller JW, Finn CT. Family, twin and adoption studies of bipolar disorder. Am J Med Genet C Semin Med Genet. 2003;123C:48-58.

12 Sullivan PF, Kendler KS, Neale MC. Schizophrenia as a complex trait: evidence from a meta-analysis of twin studies. Arch Gen Psychiatry. 2003;60:187-92

13 Craddock N, Sklar P. Genetics of bipolar disorder: successful start to a long journey. Trends Genet. 2009;25:99-105.

14 Kasap M, Rajani V, Rajani J, Dwyer DS. Surprising conservation of schizophrenia risk genes in lower organisms reflects their essential function and the evolution of genetic liability. Schizophr Res. 2018;202:120-8.

15 Millar JK, Wilson-Annan JC, Anderson S, Christie S, Taylor MS, Semple CA, et al. Disruption of two novel genes by a translocation co-segregating with schizophrenia. Hum Mol Genet. 2000;9:1415-23

16 Verkerk AJ, Matthews CA, Joosse M, Eussen BH, Heutink P, Oostra BA, Tourette Syndrome Association International Consortium for Genetics. CNTNAP2 is disrupted in a family with Gilles de la Tourette syndrome and obsessive compulsive disorder. Genomics. 2003;82:1-9.

17 The International Schizophrenia Consortium. Common polygenic variation contributes to risk of schizophrenia and bipolar disorder. Nature. 2009;460:748-52.
18 Lee SH, DeCandia TR, Ripke S, Yang J. Estimating the proportion of variation in susceptibility to schizophrenia captured by common SNPs. Nat Genet. 2012;44:247-50.

19 Nurnberger JI, Koller DL, Jung J, Edenberg HJ, Foroud T, Guella I, et al. Identification of pathways for bipolar disorder. A meta-analysis. JAMA Psychiatry. 2014;71:657-64.

20 Hyde CL, Nagle MW, Tian C, Chen X, Paciga SA, Wendland JR, et al. Identification of 15 genetic loci associated with risk of major depression in individuals of European descent. Nat Genet. 1026;48:1031-6.

21 Cristino AS, Williams SM, Hawi Z, An JY, Bellgrove MA, Schwartz CE, et al. Neurodevelopmental and neuropsychiatric disorders represent an interconnected molecular system. Mol Psychiatry. 2014;19:294-301.

$22 \mathrm{Xu} \mathrm{B}$, Roos JL, Levy S, van Rensburg EJ, Gogos JA, Karayiorgou M. Strong association of de novo copy number mutations with sporadic schizophrenia. Nat Genet. 2008;40:880-5.

23 Georgieva L, Rees E, Moran JL, Chambert KD, Milanova V, Craddock N, et al. De novo CNVs in bipolar affective disorder and schizophrenia. Hum Mol Genet. 2014; 23:6677-83.

24 Insel TR. Brain somatic mutations: the dark matter of psychiatric genetics. Mol Psychiatry. 2014;19:156-8.

25 McConnell MJ, Moran JV, Abyzov A, Akbarian S, Bae T, Cortes-Ciriano I, et al. Intersection of diverse neuronal genomes and neuropsychiatric disease: The Brain Somatic Mosaicism Network. Science. 2017;356:eaal1641.

26 Poduri A, Evrony GD, Cai X, Walsh CA. Somatic mutation, genomic variation, and neurological disease. Science. 2013;341:1237758.

27 Lodato MA, Rodin RE, Bohrson CL, Coulter ME, Barton AR, Kwon M, et al. Aging and neurodegeneration are associated with increased mutations in single human neurons. Science. 2018;359:555-9.

28 Wray NR, Visscher PM. Narrowing the boundaries of the genetic architecture of schizophrenia. Schizophr Bull. 2010;36:1423.

29 Sullivan PF, Geschwind DH. Defining the genetic, genomic, cellular and diagnostic architectures of psychiatric disorders. Cell. 2019; 177:162-83

30 Eigen M. Molecular self-organization in the early stages of evolution. Q Rev Biophys. 1971;4:149-212.

31 Dwyer DS. Amino acid sequence homology between ligands and their receptors: potential identification of binding sites. Life Sci. 1989; 45:421-9.

32 Vasas V, Fernando C, Santos M, Kauffman S, Szathmáry E. Evolution before genes. Biol Direct. 2012;7:1

33 Blalock JE, Smith EM. Hydropathic anticomplementarity of amino acids based on the genetic code. Biochem Biophys Res Comm. 1984;121:203-7.
34 Dwyer DS. Assembly of exons from unitary transposable genetic elements: implications for the evolution of protein-protein interactions. J Theor Biol. 1998;194:11-27.

35 Richardson SR, Morell S, Faulkner GJ. L1 retrotransposons and somatic mosaicism in the brain. Annu Rev Genet. 2014;48:1-27.

36 Mills RE, Bennett EA, Iskow RC, Devine SE. Which transposable elements are active in the human genome? Trends Genet. 2007;23:18391.

37 Sotero-Caio CG, Platt II RN, Suh A, Ray DA. Evolution and diversity of transposable elements in vertebrate genomes. Genome Biol Evol. 2017;9:161-77.

38 Jordan IK, Rogozin IB, Glazko GV, Koonin EV. Origin of a substantial fraction of human regulatory sequences from transposable elements. Trends Genet. 2003;19:68-72.

39 Hennah W, Thomson P, McQuillin A, Bass N, Loukola A, Anjorin A, et al. DISC1 association, heterogeneity and interplay in schizophrenia and bipolar disorder. Mol Psychiatry. 2009; 14:865-73.

40 Hooper SD, Johansson AC, Tellgren-Roth C, Stattin EL, Dahl N, Cavelier L, et al. Genomewide sequencing for the identification of rearrangements associated with Tourette syndrome and obsessive-compulsive disorder BMC Med Genet. 2012;13:123.

41 Ohno S, Wolf U, Atkin NB. Evolution from fish to mammals by gene duplication. Hereditas. 1968;59:169-87.

42 Eichler E. Recent duplication, domain accretion and the dynamic mutation of the human genome. Trends Genet. 2001;17:661-9.

43 Johnson ME, Cheng Z, Morrison VA, Scherer S, Ventura M, Gibbs RA, et al. Recurrent duplication-driven transposition of DNA during hominid evolution. Proc Natl Acad Sci U S A. 2006;103:17626-31.

44 Kurahashi $\mathrm{H}$, Inagaki $\mathrm{H}$, Ohye $\mathrm{T}$, Kogo $\mathrm{H}$, Kato T, Emanuel BS. Palindrome-mediated chromosomal translocations in humans. DNA Repair. 2006;5:1136-45.

45 Feschotte C. Transposable elements and the evolution of regulatory networks. Nat Rev Genet. 2008;9:397-405.

46 Renkawitz J, Lademann CA, Jentsch S. Mechanisms and principles of homology search during recombination. Nat Rev Mol Cell Biol. 2014;15:369-82.

47 Greene EC. DNA sequence alignment during homologous recombination. J Biol Chem. 2016;291:11572-9.

48 Kent TV, Uzunovic J, Wright SI. Coevolution between transposable elements and recombination. Phil Trans RSoc B. 2017;372:20160458.

49 Ostberg CO, Hauser L, Pritchard VL, Garza JC, Naish KA. Chromosome rearrangements, recombination suppression, and limited segregation distortion in hybrids between Yellowstone cutthroat trout (Oncorhynchus clarkia bouvieri) and rainbow trout (O. mykiss). BMC Genomics. 2013;14:570. 
50 Smith AV, Thomas DJ, Munro HM, Abecasis GR. Sequence features in regions of weak and strong linkage disequilibrium. Genome Res. 2005; 15:1519-34.

51 Myers S, Bottolo L, Freeman C, McVean G, Donnelly P. A fine-scale map of recombination rates and hotspots across the human genome. Science. 2005;310:321-4.

52 Dixon JR, Selvaraj S, Yue F, Kim A, Shen Y, $\mathrm{Hu} \mathrm{M}$, et al. Topological domains in mammalian genomes identified by analysis of chromatin interactions. Nature. 2012;485:376-80.

53 Jabbari K, Wirtz J, Rauscher M, Wiehe T. A common genomic code for chromatin architecture and recombination landscape. PloS One. 2019;14:e213278.

54 Charlesworth B. The effects of deleterious mutations on evolution at linked sites. Genetics. 2012;190:5-22.

55 Hussin JG, Hodgkinson A, Idaghdour Y, Grenier JC, Goulet JP, Gbeha E, et al. Recombination affects accumulation of damaging disease-associated mutations in human populations. Nat Genet. 2015;47:400-4.

56 Pardiñas AF, Holmans P, Pocklington AJ, Escott-Price V, Ripke S, Carrera N, et al. Common schizophrenia alleles are enriched in mutation-intolerant genes and in regions under strong background selection. Nat Genet. 2018;50:381-9.

57 McVean GAT, Charlesworth B. The effects of Hill-Robertson interference between weakly selected mutations on patterns of molecular evolution and variation. Genetics. 2000; 155:929-44.

58 Huxley J, Mayr E, Osmond H, Hoffer A. Schizophrenia as a genetic morphism. Nature. 1964;204:220-1.

59 Jarvik LF, Deckard BS. The Odyssean personality. A survival advantage for carriers of genes predisposing to schizophrenia. Neuropsychobiology. 1977;3:179-91.

60 Boyle EA, Li YI, Pritchard JK. An expanded view of complex traits: from polygenic to omnigenic. Cell. 2017;169:1177-86.

61 McVean G. What drives recombination hotspots to repeat DNA in humans? Phil Trans R Soc B. 2010;365:1213-8.

62 Gonzalez-Perez A, Sabarinathan R, Lopez-Bigas $\mathrm{N}$. Local determinants of the mutational landscape of the human genome. Cell. 2019; 177:101-14

63 Emanuel BS. Molecular mechanisms and diagnosis of chromosome 22q11.2 rearrangements. Dev Disabil Res Rev. 2008;14:11-8.

64 Schizophrenia Working Group of the Psychiatric Genomics Consortium. Biological insights from 108 schizophrenia-associated genetic loci. Nature. 2014;511:421-7.
65 Burns KH, Boeke JD. Human transposon tectonics. Cell. 2012;149:740-52.

66 Odemis S, Tuzun E, Gulec H, Semiz UB, Dasdemir S, Kucuk M, et al. Association between polymorphisms of DNA repair genes and risk of schizophrenia. Genet Test Mol Biomarkers. 2016;20:11-7.

67 Raza MU, Tufan T, Wang Y, Hill C, Zhu MY. DNA damage in major psychiatric diseases. Neurotox Res. 2016;30:251-67.

68 Akbarian S. Epigenetic mechanisms in schizophrenia. Dialogues Clin Neurosci. 2014;16:405-17.

69 Geisheker M, Haymann G, Wang T, Coe BP, Turner TN, Stessman HA, et al. Hotspots of missense mutation identify neurodevelopmental disorder genes and functional domains. Nat Neurosci. 2017;20:1043-51.

70 Alt FW, Schwer B. DNA double-strand breaks as drivers of neural genomic change, function and disease. DNA Repair. 2018;71:158-63.

71 Baillie JK, Barnett MW, Upton KR, Gerhardt DJ, Richmond TA, De Sapio F, et al. Somatic transposition alters the genetic landscape of the human brain. Nature. 2011;479:534-7.

72 Bundo M, Toyoshima M, Okada Y, Akamatsu W, Ueda J, Nemoto-Miyauchi T, et al. Increased $\mathrm{L} 1$ retrotransposition in the neuronal genome in schizophrenia. Neuron. 2014;81: 306-13.

73 Chen JM, Chuzhanova N, Stenson PD, Ferec C, Cooper DN. Meta-analysis of gross insertions causing human genetic disease: mutational mechanisms and the role of replication slippage. Hum Mutat. 2005;25:207-21.

74 Heng HHQ. The genome-centric concept: resynthesis of evolutionary theory. BioEssays. 2009;31:512-25.

75 Finn EH, Misteli T. Molecular basis and biological function of variability in spatial genome organization. Science. 2019;365:eaaw9498.

76 Kasap M, Dwyer DS: How genetic effect size and gene interactions shape the genetic architecture of schizophrenia. 2019. Submitted for publication.

77 Koch E, Ristroph M, Kirkpatrick M. Long range linkage disequilibrium across the human genome. PLoS One. 2013;8:e80754.

78 Gelbart WM. Synapsis-dependent allelic complementation at the decapentaplegic gene complex in Drosophila melanogaster. Proc Natl Acad Sci U S A. 1982;79:2636-40.

79 Dillon N, Trimborn T, Strouboulis J, Fraser P, Grosveld F. The effect of distance on longrange chromatin interactions. Mol Cell. 1997; 1:131-9.
80 Dekker J, Rippe K, Dekker M, Kleckner N. Capturing chromosome conformation. Science. 2002;295:1306-10.

81 Lee GR, Spilianakis CG, Flavell RA. Hypersensitive site 7 of the TH2 locus control region is essential for expressing TH2 cytokine genes and for long-range intrachromosomal interactions. Nat Immunol. 2005;6:42-8

82 Lomvardas S, Barnea G, Pisapia DJ, Mendelsohn M, Kirkland, AR. Interchromosomal interactions and olfactory receptor choice. Cell. 2006;126:403-13.

83 Lieberman-Aiden E, van Berkum NL, Williams L, Imakaev M, Ragoczy T, Telling A, et al. Comprehensive mapping of long-range interactions reveals folding principles of the human genome. Science. 2009;326:289-93.

84 Maass PG, Barutcu AR, Rinn JL. Interchromosomal interactions: a genomic love story of kissing chromosomes. J Cell Biol. 2018; 218:27-38.

85 Won H, de la Torre-Ubieta L, Stein JL, Parikshak NN, Huang J, Opland CK, et al. Chromosome conformation elucidates regulatory relationships in developing human brain. $\mathrm{Na}-$ ture. 2016;538:523-7.

86 Whalen S, Pollard KS. Most chromatin interactions are not in linkage disequilibrium. Genome Res. 2019;29:334-43.

87 Kikuta H, Laplante M, Navratilova P, Komisarczuk AZ, Engstrom PG, Fredman D, et al. Genomic regulatory blocks encompass multiple neighboring genes and maintain conserved synteny in vertebrates. Genome Res. 2007; 17:545-55.

88 Kirk IK, Weinhold N, Brunak S, Belling K. The impact of the protein interactome on the syntenic structure of mammalian genomes. PLoS One. 2017;12:e0179112.

89 Hall RM, Collis CM. Mobile gene cassettes and integrons: capture and spread of genes by site-specific recombination. Mol Microbiol. 1995; 15:593-600.

90 Barzel A, Kupiec M. Finding a match: how do homologous sequences get together for recombination? Nat Rev Genet. 2008;9:27-37.

91 Zwang TJ, Tse ECM, Barton JK. Sensing DNA through DNA charge transport. ACS Chem Biol. 2018;13:1799-809.

92 Kurian P, Dunston G, Lindesay J. How quantum entanglement in DNA synchronizes double-strand breakage by type II restriction endonucleases. J Theor Biol. 2016;391:10212.

93 Hernando-Herraez I, Garcia-Perez R, Sharp AJ, Marques-Bonet T. DNA methylation: insights into human evolution. PLoS Genet. 2015;11:e1005661. 